\title{
Die Bone and Joint Decade - Chancen für Orthopädie und Unfallchirurgie
}

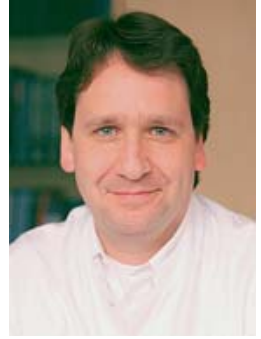

K. E. Dreinhöfer

Bibliografie

DOI 10.1055/s-2007-965634 Z Orthop Unfall 2007; 145: 399-402 @ Georg Thieme Verlag KG Stuttgart • New York • ISSN 1864-6697

Korrespondenzadresse Dr. med. Karsten E. Dreinhöfer Orthopädische Universitätsklinik Ulm am RKU Oberer Eselsberg 45 $89081 \mathrm{Ulm}$

Tel.: 0731/177-1114 Fax: 0731/177-1118

karsten.dreinhoefer@ uni-ulm.de
Muskuloskeletale Erkrankungen und Verletzungen sind weltweit die Hauptursache für chronische Schmerzen und körperliche Behinderungen, zudem haben diese Krankheitsbilder eine enorme gesellschaftliche und volkswirtschaftliche Bedeutung - und dennoch fehlte bisher die notwendige Aufmerksamkeit in der Ärzteschaft, im öffentlichen Bewusstsein und in der politischen Diskussion bei der Priorisierung von Gesundheitsleistungen. Diese Krankheitsbilder führen nur selten unmittelbar zum Tode. Nachdem aber insbesondere dieses Kriterium in der Vergangenheit die Bedeutung eines Krankheitsbereiches in der Öffentlichkeit bedingt hat, wurden muskuloskeletale Erkrankungen häufig als unvermeidbare altersbedingte Erscheinungen und Verletzungen als unvermeidbare Folgen von Unfällen angesehen. Erst die sich langsam verändernde vermehrte Priorisierung von Lebensqualität vor dem Tode als Gesundheitsziel, mit der Betonung einer schmerzfreien Mobilität und Partizipation am sozialen Leben, ermöglicht es die Schwerpunktsetzung bei der Prävention und Gesundheitsversorgung zu beeinflussen.

Die Weltgesundheitsorganisation (WHO) und die Vereinten Nationen (UNO) haben die Bedeutung chronischer nicht-letaler Erkrankungen erkannt und die Bone and Joint Decade ausgerufen, um die Lebensqualität von Menschen mit muskuloskeletalen Problemen weltweit zu verbessern allerdings ohne selbst zunächst wesentliche finanzielle oder logistische Ressourcen bereitzustellen $[1,2]$.

Eine erste internationale Konsensuskonferenz mit Vertretern von 50 wissenschaftlichen Fachgesellschaften und Patientenorganisationen konnte die vorliegenden Kenntnisse über Ausmaß und Bedeutung der muskuloskeletalen Erkrankungen und Verletzungen zusammenstellen, die vorrangigen Probleme aufzeigen und Handlungsempfehlungen geben [3]. Die gemeinsamen Bemühungen sollten darauf abzielen, die Lebensqualität der Menschen mit muskuloskeletalen Erkrankungen und Verletzungen zu verbessern durch:

- ein wachsendes Bewusstsein für die Bedeutung dieser Probleme in der Gesellschaft,

- eine Darstellung und Förderung des Präventionspotenzials dieser Erkrankungen,

- eine Einbeziehung der Patienten in die Entscheidungsprozesse bei der Gesundheitsversorgung.

Die Optimierung der Prävention, Diagnostik und Behandlung von muskuloskeletalen Erkrankungen und Verletzungen sowie die Reduktion der hohen indirekten Krankheitskosten sind nur möglich durch

- eine gesteigerte Forschungsförderung,

- eine verbesserte und intensivierte Ausbildung der Medizinstudenten,

- eine verbesserte, zeitgerechte und kompetent abgestufte Diagnostik und Therapie.

Zwischenzeitlich haben weltweit mehr als 750 wissenschaftliche Gesellschaften und Patientenorganisationen die Ziele der Bone and Joint Decade offiziell unterstützt, in 95 Ländern sind nationale Koordinatoren benannt worden, in 65 Ländern haben sich interdisziplinäre Arbeitskreise (nationale Netzwerke) gebildet, bestehend aus den relevanten ärztlichen Verbänden und Patientenorganisationen, um gemeinsam die nationalen Aktivitäten der Dekade zu koordinieren und auf die lokalen Bedürfnisse abzustimmen [4]. Das „Deutsche Netzwerk der Bone and Joint Decade" ist die nationale Dachorganisation für alle mit Erkrankungen und Verletzungen der Haltungs- und Bewegungsorgane befassten wissenschaftlichen Gesellschaften, Berufsverbände und Patientenorganisationen und umfasst zurzeit 21 Mitgliedsgesellschaften (www.boneandjointdecade.de).

Auf den verschiedenen internationalen Ebenen werden Aktivitäten unterschiedlichster Art zur Verfolgung der Ziele der Bone and Joint Decade angestrengt [5]:

Basierend auf einer interdisziplinären Zusammenarbeit von mehr als 150 Experten konnte ein Technical Report der WHO unter dem Titel „The Burden of Musculoskeletal Conditions at the Start of the New Millennium“ publiziert werden, in dem erstmals weltweite Daten zu Inzidenz und Prävalenz muskuloskeletaler Erkrankungen und Verletzungen, sowie Instrumente zur Bestimmung des Gesundheitszustandes zusammengestellt worden sind [6]. Dieser Bericht offenbarte jedoch auch noch erhebliche Kenntnisdefizite in vielen Regionen, aber auch Krankheitsbereichen.

Im Rahmen des „Bone and Joint Decade Monitor Projects“ werden Strategien zur Verbesserung der gesundheitsbezogenen Lebensqualität für Menschen mit Erkrankungen des Haltungs- und Bewegungsapparates entwickelt, basierend auf der Evaluation effektiver Präventions- und Therapiestrategien, sowie der Analyse der gegenwärtigen und der „optimalen“ Versorgungssituation (Standards of Care), sowie der Kosten und der Prioritäten $[7,8]$.

Das „European Bone and Health Strategies Project" hat mit Unterstützung der Europäischen 
Kommission Risikofaktoren für muskuloskeletale Erkrankungen und Verletzungen identifiziert und Präventionsstrategien entwickelt. In Zusammenarbeit mit Experten der europäischen wissenschaftlichen Gesellschaften (EFORT, EULAR und IOF) sowie Vertretern von Patientenorganisationen konnten evidenzbasierte Empfehlungen erarbeitet werden, die nun in den einzelnen Ländern implementiert werden sollen [9].

In einem weiteren von der Europäischen Kommission unterstützten Projekt, „Indicators for Monitoring Musculoskeletal Problems and Conditions“, wurden Indikatoren zusammengestellt, die gegenwärtig für die Analyse von Erkrankungen und Verletzungen der Haltungs- und Bewegungsorgane eingesetzt werden. Basierend auf dieser Grundlage wurden Empfehlungen zum Einsatz relevanter muskuloskeletaler Indikatoren für künftige bevölkerungsbasierte Surveys in den Mitgliedsländern konsentiert [10].

Die Bone and Joint Decade konnte gemeinsam mit anderen Partnern erreichen, dass erstmals die Vollversammlung der Vereinten Nationen das Thema Verkehrssicherheit diskutiert und entsprechende Resolutionen zur Prävention von Verkehrsunfällen verabschiedet hat [11]. Auch die G8-Staaten haben daraufhin auf ihren letzten Gipfeltreffen diese Thematik behandelt. Während in Deutschland die Unfallzahlen kontinuierlich zurückgehen, steigt die Zahl der Unfallverletzten und -toten in den Ländern mittleren und geringen Einkommens explosiv an. Die WHO wurde deshalb aufgefordert diese Problematik fokussiert anzugehen, indem gezielt sektorenübergreifende Präventionsprogramme implementiert werden. Unter dem Motto „Road safety is no accident" werden weltweit Aufklärungskampagnen mit dem Ziel veranstaltet, die Zahl der Toten und Verletzten im Straßenverkehr zu reduzieren [12].

Aufgrund der weltweiten Diskrepanz zwischen volkswirtschaftlicher Bedeutung und öffentlicher Forschungsförderung bemüht sich die BJD gegenwärtig in Europa zusammen mit den wissenschaftlichen Gesellschaften EFORT und EULAR um eine Priorisierung der muskuloskeletalen Forschung im Rahmen des neuen europäischen Forschungsrahmenprogramms (www.cordis.europa.eu/fp7) [13], in den USA sind durch die Anstrengungen der BJD schon erste Erfolge zu verzeichnen gewesen [14].

Eine weltweite Analyse des Lehrumfanges an den medizinischen Fakultäten zeigte einen erschreckend geringen muskuloskeletalen Ausbildungsumfang [15]. Die Bone and Joint Decade Musculoskeletal Education Task Force, eine internationale, multidisziplinäre Arbeitsgruppe, erarbeitete daraufhin ein Kernkurrikulum, das die notwendigen Grundkenntnisse eines jeden Arztes definiert und nun an den einzelnen Fakultäten implementiert werden soll [16].

In Deutschland nutzen die verschiedenen Fachgesellschaften und Organisationen die Möglichkeiten der „Plattform BJD“ und der interdisziplinären Zusammenarbeit sehr unterschiedlich. Während einige diese Chancen bisher nur gering wahrgenommen haben, ist von anderen Gesellschaften die Bone and Joint Decade publikumswirksam für nationale Jahreskongresse, aber auch von Verbänden oder Kliniken für nationale Aktivitäten oder lokale Veranstaltungen genutzt worden. Daneben konnten einige Aktivitäten zur besseren Bewusstmachung der Erkrankungen und Verletzungen, aber auch ihrer Prävention und Therapie, in der Öffentlichkeit initiiert werden. Hierzu zählen neben vom BMGS finanzierte Journalistenseminare und Artikel, die in der Fach- und Publikumspresse veröffentlicht wurden, auch spezifische Aufklärungskampagnen, so z. B. „Orthopädie bewegt“.
Um die Bedeutung der muskuloskeletalen Erkrankungen und Verletzungen für die Gesellschaft überzeugend darzustellen, bedarf es belastbarer Daten zu Inzidenzen und Prävalenzen der Erkrankungen, zu den krankheitsbedingten Einschränkungen und Folgen, sowie zu den direkten und indirekten Kosten. Im Rahmen eines vom Bundesgesundheitsministerium geförderten Forschungsprojekts [17] konnten umfangreiche Datenanalysen vorgenommen werden, die erstmals einen Eindruck der muskuloskeletalen Krankheitslast (Burden of Disease) in Deutschland erlauben:

- Muskuloskeletale Erkrankungen und Verletzungen sind die Hauptursache für chronische Behinderungen und Einschränkungen in der Gesellschaft. 75\% der Deutschen hatten in den letzten 12 Monaten Schmerzen der Haltungs- und Bewegungsorgane. Jeder 5. Deutsche war in den letzten 12 Monaten beim Orthopäden, bei den über 50-Jährigen war es jede 3. Frau und jeder 4. Mann.

- Muskuloskeletale Erkrankungen führten zu mehr als 1,3 Mio. Krankenhausbehandlungen, Verletzungen zu weiteren 1,6 Mio.; mit knapp 10\% bzw. $8 \%$ aller Krankenhausfälle waren sie damit die viert- bzw. fünfthäufigste Diagnosegruppe im stationären Bereich. Jede 3. stationäre Operation wurde an den Haltungs- und Bewegungsorganen vorgenommen.

- Seit Jahren stehen Erkrankungen der Haltungs- und Bewegungsorgane an der Spitze des Arbeitsunfähigkeitsgeschehens, mit $18 \%$ aller AU-Fälle und $27 \%$ aller AU-Tage, Verletzungen verursachten weitere $9 \%$ bzw. $13 \%$.

- Insgesamt sind durch muskuloskeletale Erkrankungen (25,2 Mrd. Euro) und Verletzungen (10,6 Mrd. Euro) in Deutschland im Jahr 2002 direkte Kosten in Höhe von mindestens 36 Mrd. Euro entstanden. Dies entspricht bereits mehr als 1,7\% des gesamten Bruttosozialproduktes und 16\% der gesamten Krankheitskosten. Unter Berücksichtigung der Produktionsausfallskosten (zusammen 40\% der verlorenen Erwerbstätigkeitsjahre) können mehr als $100 \mathrm{Mrd}$. Euro angesetzt werden, entsprechend 4,8\% des Bruttonationaleinkommens.

Basierend auf Ergebnissen aus diesem Forschungsprojekt wird das vorliegende Heft der „Zeitschrift für Orthopädie und Unfallchirurgie“ mit einer ersten differenzierten Arbeit zu der sozialmedizinischen Bedeutung der degenerativen Gelenkerkrankungen eingeleitet.

Den Gelenkerkrankungen kommt bei den muskuloskeletalen Erkrankungen mit 10,6 Mrd. Euro bzw. 42,1\% die größte Bedeutung bei den direkten Krankheitskosten zu. Drei Fünftel der Ausgaben für die degenerativen Gelenkerkrankungen treten im stationären Sektor auf (4,2 Mrd. Euro), davon 3,0 Mrd. Euro Krankenhauskosten (71\%) sowie weitere große Kostenblöcke im Bereich der stationären Rehabilitation (720 Mio. Euro) und der stationären Pflege (490 Mio. Euro). Im ambulanten Sektor nehmen die ärztlichen Behandlungskosten etwa ein Drittel der Kosten (740 Mio. Euro) ein. Mit 5,0 Mrd. Euro fallen fast 70\% der Kosten für degenerative Gelenkerkrankungen bei Frauen an. Von diesen Erkrankungen sind überwiegend (96\%) die über 45-Jährigen betroffen, wobei der Schwerpunkt mit 4,1 Mrd. Euro zwischen dem 65. und 85. Lebensjahr lag.

Wenn man bedenkt, dass die Arthrosen zu den häufigsten Ursachen für den Krankenstand der berufstätigen Gesellschaft zählen, erscheint die Notwendigkeit der Primärprävention ebenso wie die adäquate, stadiengerechte Therapie eindeutig. Betrachtet man allerdings die deutliche Diskrepanz zwischen Beschwerde- und Behandlungsprävalenz, sind offensichtlich unterschiedliche Einschätzungen der Indikation zur Hüftendoprothetik zwi- 
schen Operateuren und Zuweisern [18], aber insbesondere auch ein mangelnder Informationsstand der Patienten [19] verantwortlich. Hier scheint noch ein großer Klärungs- und Informationsbedarf zu existieren - der auch im Rahmen der Bone and Joint Decade interdisziplinär und sektorenübergreifend angegangen werden sollte.

Auch in anderen Schwerpunktbereichen der Bone and Joint Decade hat es in Deutschland Aktivitäten gegeben. So wurde der muskuloskeletale Lehrumfang in Deutschland untersucht - und erwies sich mit etwa $5 \%$ ebenfalls als absolut ungenügend, um die Thematik des gesamten Gebietes kompetent und ausreichend den Medizinstudenten zu vermitteln [20]. Unter Berücksichtigung des globalen BJD-Kurrikulums [21] erarbeitet gegenwärtig eine orthopädisch-unfallchirurgische Arbeitsgruppe die Inhalte eines Lernzielkataloges für das neue gemeinsame Fach. Mittelfristig sollte überlegt werden, dies dann auch mit anderen betroffenen Fächern aus dem muskuloskeletalen Bereich abzugleichen, um ein muskuloskeletales Kurrikulum in der ganzen Breite anbieten zu können.

Der Umfang der Forschungsförderung orientiert sich in Deutschland bisher am internationalen Standard und entspricht nicht der Bedeutung der muskuloskeletalen Erkrankungen und Verletzungen. In einer aktuellen Untersuchung zeigt sich, dass gerade einmal 2,8\% der jährlichen Forschungsförderung durch BMBF und DFG für muskuloskeletale Fragestellungen aufgewandt wird (persönliche Mitteilung Prof. Eulert/Prof. Jakob). Allerdings scheint berechtigte Hoffnung zu bestehen, dass in der aktuellen Diskussion des „Road Map“-Prozesses zur Definition der prioritären Forschungsgebiete in Deutschland die muskuloskeletale Thematik vermehrt berücksichtigt wird.

Auch das aktuelle politische Geschehen erfordert eine Intensivierung der interdisziplinären, sektorübergreifenden Zusammenarbeit in Kooperation mit den Patienten. So spricht der aktuelle Bericht des Sachverständigenrates [22] über „Angemessenheit und Verantwortlichkeit in der Gesundheitsversorgung“ und statuiert, dass „eine zielorientierte Gesundheitsversorgung auf einem Nutzenkonzept (basiert), das über die absolute Wirksamkeit hinaus Patienten- und gesellschaftliche Präferenzen einbezieht. Der Begriff der Angemessenheit macht die Determinanten der relativen Wirksamkeit für Allokationsfragen zugänglich...“ Diese Zielrichtung hat das Potenzial eine enorme Diskussion und Verteilungskämpfe zwischen den Gebieten zu entfachen. Hier sind die wissenschaftlichen Gesellschaften und Berufsverbände gemeinsam gefordert, zeitnah eine adäquate Evaluation der muskuloskeletalen Versorgungsmaßnahmen im Rahmen von Versorgungsforschungsprojekten vorzunehmen, um selbst kompetente Argumentationsgrundlagen vorweisen zu können [23].

Die Aktivitäten der Bone and Joint Decade werden auch nach dem Jahr 2010 weitergehen und ausgebaut werden müssen. Die „Brain Decade“ der 90er-Jahre hat eindrücklich gezeigt, dass erste Ergebnisse frühestens am Ende der Dekade zu erwarten sind, und dass die strukturierte Fortführung im zweiten Jahrzehnt es dann ermöglicht, tatsächliche Fortschritte zu erzielen. In der strategischen Planung ist eine vermehrte Regionalisierung der BJD-Aktivitäten vorgesehen, um hier durch gemeinsame Aktivitäten z.B. auf europäischer Ebene (European Council) den sich verändernden politischen Entscheidungswegen zu folgen. Erste Kooperationen zwischen EFORT, EULAR und Patientenorganisationen sind der beste Beweis, dass durch ein breit aufgestelltes Bündnis die Ziele der Bone and Joint Decade am besten zu erreichen sind. Es wäre wünschenswert, diese gemeinsamen Aktivitäten auch auf deutscher Ebene zu intensivieren!

So können die zwischenzeitlich zusammengestellten Daten und Fakten als Grundlage dienen, die Bedeutung der muskuloskeletalen Erkrankungen und Verletzungen gemeinsam mit den anderen beteiligten Fachgesellschaften und Patientenorganisationen auf dem Boden der Bone and Joint Decade deutlich zu betonen. Anschließend muss die daraus resultierende Priorisierung der Ressourcenallokation in allen Bereichen artikuliert und eingefordert werden. Das gilt sowohl für den Umfang der Lehre an den Hochschulen, als auch für das Ausmaß der Forschungsförderung - aber insbesondere auch für die Versorgungsmöglichkeiten muskuloskeletaler Krankheiten in Praxis und Klinik!

\section{Literatur}

1 Dreinhöfer KE. Bone and Joint Decade 2000-2010: Prävention und Management effizienter gestalten. Deutsches Ärzteblatt 2000; 97: A3478-A3481

2 Niethard FU, Puhl W. Die „Bone and Joint Decade 2000 to 2010“. Z Orthop Ihre Grenzgeb 1999; 137: 1

3 The Bone and Joint Decade 2000-2010 for prevention and treatment of musculo-skeletal disorders. Lund, Sweden, April 17 -18, 1998. Proceedings. Acta Orthop Scand (Suppl) 1998; 281: 1 - 86

4 Lidgren L. The Bone and Joint Decade 2000-2010. Bull World Health Organ 2003; 81: 629

5 Dreinhöfer K. Bone and Joint Decade - die zweite Halbzeit ist angebrochen. Orthopädie Mitteilungen 2006; 2: 98 - 101

6 WHO Scientific Group on the Burden of Musculoskeletal Conditions at the Start of the New Millennium. The burden of musculoskeletal conditions at the start of the new millennium. World Health Organ Tech Rep Ser 2003; 919: 1 - 218

7 Woolf $A D$. The bone and joint decade. Strategies to reduce the burden of disease: the Bone and Joint Monitor Project. J Rheumatol (Suppl) 2003; 67: 6-9

8 Woolf $A D$, Akesson $K$. Understanding the burden of musculoskeletal conditions. The burden is huge and not reflected in national health priorities. BMJ 2001; 322: 1079-1080

9 European Bone and Joint Health Strategies Project. European Action Towards Better Musculoskeletal Health - A Public Health Strategy to Reduce the Burden of Musculoskeletal Conditions. Lund: The Bone \& Joint Decade, 2004

10 Indicators for Monitoring Musculoskeletal Problems and Conditions. Musculoskeletal Problems and Functional Limitation. The Great Public Health Challenge for the 21st Century. In: European Commission - Directorate General Public Health (Ed). Lund: The Bone \& Joint Decade, 2003

11 United Nations General Assembly. Improving global road safety. Fiftyeighth session - global road safety crisis. New York, 2004

12 World Health Organization. World report on road traffic injury prevention. Geneva: World Health Organization, 2004

13 Kvien TK. A good year for European rheumatology. Ann Rheum Dis 2007; 66: 1

$14 \mathrm{Katz}$ SI. The national bone and joint decade: impact for rheumatic diseases. Arthritis Rheum 2005; 52: $707-709$

15 Akesson K, Dreinhöfer KE, Woolf AD. Improved education in musculoskeletal conditions is necessary for all doctors. Bull World Health Organ 2003; 81: $677-683$

16 Woolf $A D$, Walsh NE, Akesson K. Global core recommendations for a musculoskeletal undergraduate curriculum. Ann Rheum Dis 2004; 63: $517-524$

17 Dreinhöfer K, Merx H, Puhl W. Muskuloskelettale Erkrankungen und Verletzungen. Gesundheitsberichterstattung des Bundes. Berlin: Robert Koch-Institut, im Review

18 Dreinhöfer KE, Dieppe P, Sturmer T et al. Indications for total hip replacement: comparison of assessments of orthopaedic surgeons and referring physicians. Ann Rheum Dis 2006; 65: 1346-1350

19 Hawker GA. Who, when, and why total joint replacement surgery? The patient's perspective. Curr Opin Rheumatol 2006; 18: 526-530

20 Dreinhöfer K, Stöfele, T, Puhl, W. Erhalten Studenten an deutschen Universitäten genügend Unterricht in Orthopädie? Zeitschrift für Orthopädie 2003; 141: S29 
21 Dreinhöfer K. Musculoskelettales Lehrcurrikulum. Orthopädie Mitteilungen 2006; 3: 196 - 199

22 Sachverständigenrat zur Begutachtung der Entwicklung im Gesundheitswesen. Kooperation und Verantwortung - Voraussetzungen einer zielorientierten Gesundheitsversorgung, 2007
23 Dreinhöfer K, Dieppe P, Flören M et al. Muskuloskelettale Versorgungsforschung - Beispiele zum aktuellen Kenntnisstand und offene Fragen. In: Hey M, Maschewsky-Schneider U, Busse R et al. (Hrsg). Kursbuch Versorgungsforschung. Berlin: Medizinisch Wissenschaftliche Verlagsgesellschaft, 2006: 58-69 